\title{
LIGADURA VIDEOLAPAROSCÓPICA DA ARTÉRIA MESENTÉRICA INFERIOR COMO TRATAMENTO DE ENDOLEAK PERSISTENTE APÓS CORREÇÃO ENDOVASCULAR DE ANEURISMA DE AORTA ABDOMINAL
}

\author{
VIDEOLAPAROSCOPIC LIGATION OF INFERIOR MESENTERIC ARTERY AS A \\ TREATMENT OF PERSISTENT ENDOLEAK AFTER ENDOLUMINAL ABDOMINAL \\ AORTIC ANEURYSM REPAIR
}

\author{
Gaudencio Espinosa (TCBC-RJ) ${ }^{1}$ \\ Delta Madureira (TCBC-RJ) ${ }^{1}$ \\ Warley Dias Siqueira Mendes (RCBC-RJ) ${ }^{2}$
}

\section{INTRODUÇÃO}

O tratamento endovascular do aneurisma de aorta abdominal, iniciado cerca de uma década atrás, vem sendo considerado de escolha para pacientes que apresentam aneurisma de aorta abdominal, e que sejam de alto risco para a realização da cirurgia convencional ${ }^{1}$.

A persistência ou recorrência de fluxo para o interior do saco aneurismático após o implante de uma endoprótese vascular é denominada endoleak (fuga ou vazamento $)^{1,2}$, podendo perpetuar o aumento de pressão no saco aneurismático, o qual é denominado endotensão ${ }^{2}$. A prevalência de endoleaks após correção endovascular de aneurisma é muito variável, tendo sido relatada na literatura entre $2,4 \%$ e $45,5 \%$. Sua importância clínica está diretamente relacionada com o aumento do risco de ruptura do aneurisma ${ }^{3}$.

O objetivo deste trabalho é relatar um caso de endoleak tipo II pela artéria mesentérica inferior tratado com sucesso mediante ligadura videolaparoscópica.

\section{RELATO DO CASO}

Paciente de 72 anos, sexo masculino, branco, natural do Rio de Janeiro, hipertenso, com antecedentes de hiperplasia prostática benigna e úlcera gástrica, em uso de diltiazem, atenolol, furosemida (associada com cloreto de potássio) e omeprazol.
Procurou atendimento médico em maio de 1998, com dor precordial tipo anginosa, com evolução de sete meses, de forte intensidade com irradiação para o membro superior esquerdo e parestesia do mesmo. Foi hospitalizado e durante a realização dos exames complementares verificou-se a presença de aneurisma de aorta abdominal. Tratado ainda, na mesma internação, da doença arterial coronária (de 70\%) com revascularização miocárdica, recebeu alta após recuperação clínica.

Tratando-se de um paciente de risco cirúrgico elevado, foi indicado o tratamento endovascular do aneurisma com a realização prévia de arteriografia da aorta abdominal com cateter centimetrado, para a escolha da endoprótese mais adequada. À injeção do meio de contraste foi possível identificar a origem das artérias renais, com colo infra-renal muito angulado. Foram realizados também novos exames de eco-Doppler e tomografia computadorizada visando ao tratamento do aneurisma. Os exames mostraram aneurisma de aorta abdominal infra-renal medindo $9,4 \mathrm{~cm}$ no seu maior diâmetro, acometendo a artéria ilíaca comum direita e preservando a artéria ilíaca comum esquerda (Figura 1A).

O procedimento cirúrgico endovascular foi realizado em março de 1999, sob anestesia geral, mediante a dissecção inguinal bilateral e reparo das artérias femorais e punção da artéria braquial esquerda com introdução de cateter 5 French tipo pig-tail, o qual foi posicionado na aorta torácica descendente para controle angiográfico do procedimento.

1. Professor Adjunto do Departamento de Cirurgia da Universidade Federal do Rio de Janeiro (UFRJ)

2. Médico-Residente do Serviço de Cirurgia Vascular do Hospital Universitário Clementino Fraga Filho da UFRJ.

Recebido em 18/06/2001.

Aceito para publicação em 22/11/2001.

Trabalho realizado no Departamento de Cirurgia do Hospital Universitário da Universidade Federal do Rio de Janeiro, HU-UFRJ 
Após acesso cirúrgico inguinal bilateral com dissecção, reparo e arteriotomia longitudinal das artérias femorais direita e esquerda, em anticoagulação plena com heparina na dose de $100 \mathrm{UI} / \mathrm{kg}$, foi realizada angioplastia ilíaca bilateral mediante a técnica de kissing balloon com dois cateteres-balão 10 x 40mm insuflados a uma pressão de 8 ATM, devido à doença aterosclerótica severa. Em seguida, posicionaram-se dois fios-guia Extra Stiff AMPLATZ, para posterior introdução do sistema liberador. Realizou-se o implante da endoprótese vascular tipo TALENT, em posição supra-renal (Freeflow), e posterior liberação de ambos os ramos da endoprótese para as artérias ilíacas. Uma vez realizado o implante, executou-se a reconstrução das artérias femorais, com endarterectomia e sutura contínua da parede com polipropileno 6-0.

No estudo angiográfico de controle pós-procedimento, foi observado bom posicionamento da endoprótese vascular, encontrando-se a mesma implantada de forma hermética proximal e distalmente, e artérias ilíacas com bom fluxo.

$\mathrm{Na}$ avaliação pós-operatória imediata (30 dias), o paciente apresentou trombose completa do saco aneurismático, com bom posicionamento da endoprótese vascular, e $10,8 \mathrm{~cm}$ no seu maior diâmetro.

O paciente foi acompanhado clínica e radiologicamente mediante a realização de tomografia helicoidal com contraste venoso a cada oito meses. No entanto, o controle tomográfico após oito meses identificou pequena área de vazamento na porção anterior do saco aneurismático através de fluxo retrógrado pela artéria mesentérica inferior, diagnosticando um endoleak do tipo II, e aumento do diâmetro do aneurisma para $11,4 \mathrm{~cm}$ (Figura 1B).

Inicialmente foi adotada conduta expectante, na possibilidade de trombose espontânea da artéria mesentérica inferior e consequientemente a resolução do endoleak. En- tretanto, no controle tomográfico de 16 meses observou-se um aumento do endoleak e um crescimento progressivo do aneurisma, agora medindo $12,1 \mathrm{~cm}$ no seu maior diâmetro (Figura 2A).

Foi submetido em janeiro de 2001 à cirurgia videolaparoscópica com ligadura da artéria mesentérica inferior. A operação efetuou-se sob anestesia geral, com o paciente em decúbito dorsal, o cirurgião situado do lado direito do paciente e o monitor do lado oposto. Fez-se a introdução dos trocarteres, insuflação da cavidade peritoneal com dióxido de carbono a uma pressão de $14 \mathrm{mmHg}$ e utilizada ótica de $30^{\circ}$. Após o acesso intraperitoneal, realizou-se tração ventral do cólon transverso, para retificação do meso, abertura do retroperitônio com conseqüente dissecção e posterior ligadura dupla da artéria mesentérica inferior justa-aneurisma, com polipropileno número zero. O tempo operatório foi de 110 minutos.

O paciente teve boa evolução clínica e alta hospitalar no segundo dia de pós-operatório, sendo mantido em acompanhamento ambulatorial. A realização de tomografia computadorizada helicoidal com três meses de pós-operatório evidenciou trombose completa do saco aneurismático com medida de $11,2 \mathrm{~cm}$ no seu maior diâmetro, confirmando o sucesso do tratamento do endoleak pela via videolaparoscópica (Figura 2B).

\section{DISCUSSÃO}

Os estudos de imagem realizados tanto no preparo pré-operatório quanto no acompanhamento pós-operatório de um paciente submetido ao tratamento endovascular de um aneurisma de aorta são de grande importância ${ }^{4}$.

A presença ou ausência de endoleak aos exames de imagem não podem ser consideradas critérios de falha ou sucesso do tratamento, uma vez que a endotensão também
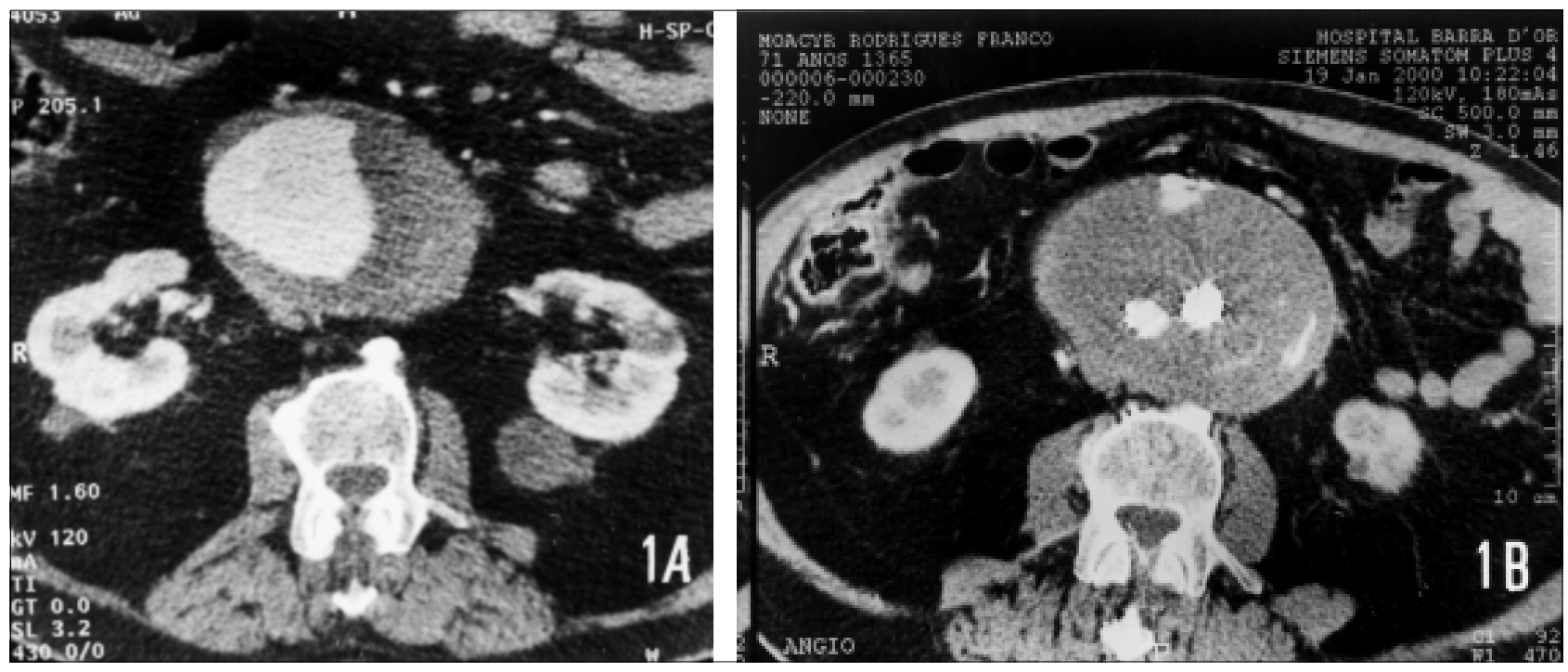

Figura $1-A)$ Corte tomográfico axial pré-operatório evidenciando aneurisma de aorta abdominal de 9,4cm. B) Observa-se no controle de oito meses, após o implante da endoprótese vascular, trombose incompleta do saco aneurismático com área de vazamento na porção anterior causada pela artéria mesentérica inferior. 

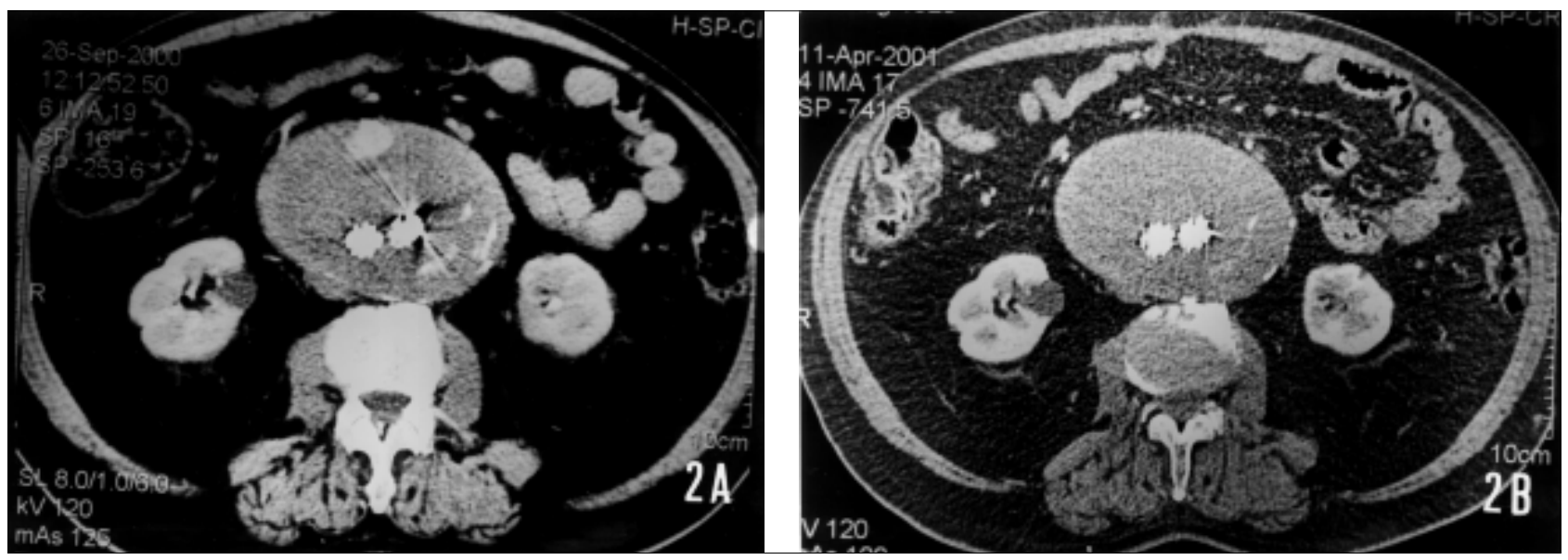

Figura 2 - A) Corte tomográfico evidenciando aumento da área de vazamento pela artéria mesentérica inferior e aumento do diâmetro do aneurisma para $12,1 \mathrm{~cm}$. B) Resultado tomográfico final, após ligadura videolaparoscópica da artéria mesentérica inferior, com trombose completa do saco aneurismático.

pode ser transmitida, sem fluxo, através de uma camada de trombo e ser causa de ruptura do aneurisma ${ }^{2}$. No nosso caso, após a realização da primeira tomografia computadorizada helicoidal, com um mês de pós-operatório, pudemos observar um aumento do aneurisma, porém não identificamos a presença de vazamento, denotando a presença de endotensão.

Os endoleaks podem ser detectados através de ecocolor-Doppler, angiografia e tomografia computadorizada, sendo a tomografia computadorizada, principalmente do tipo helicoidal, o método de diagnóstico mais sensível ${ }^{3}$.

Os endoleaks podem ser classificados em quatro ti$\operatorname{pos}^{1}$. Tipo I, resulta de um vazamento no local de fixação do enxerto; tipo II, resulta de fluxo retrógrado proveniente das artérias lombares ou da mesentérica inferior; tipo III, resulta de desconexão, desintegração ou ruptura de um componente da endoprótese; e o tipo IV, resulta do aumento da porosidade da prótese. O conhecimento da fonte de origem do endoleak é, pois, de grande importância na decisão terapêutica a ser realizada, como podem ser a embolização, colocação de stent adicional, cirurgia convencional, ou simplesmente uma conduta expectante. No nosso caso, tratava-se de um endoleak tipo II proveniente da artéria mesentérica inferior.

O aumento ou a estagnação do tamanho do aneurisma aórtico é sugestivo da presença de vazamentos para o interior do saco aneurismático, ou seja, endoleak, e conseqüente risco de ruptura ${ }^{4}$. O endoleak tipo II costuma apresentar resolução espontânea em até quatro meses, ${ }^{4}$ motivo pelo qual inicialmente adotamos uma conduta expectante.
A ocorrência de endoleaks ainda promove dúvidas em relação ao melhor método para seu tratamento, quando não solucionado espontaneamente. $\mathrm{O}$ uso de esponja gelatinosa no saco aneurismático, a embolização com molas, a cirurgia convencional e a ligadura videolaparoscópica são alguns métodos descritos para o tratamento de endoleak tipo II $^{5}$. No nosso caso, o método adotado para o tratamento do endoleak causado pela artéria mesentérica inferior foi a ligadura por via videolaparoscópica.

A dificuldade técnica encontrada para entrar e embolizar a artéria nutridora do saco aneurismático ao longo da endoprótese, por meio de um cateterismo transfemoral, parece tornar a embolização um método mais difícil de se realizar que a ligadura videolaparoscópica ${ }^{5}$. Além da intervenção ao longo da endoprótese, para a realização da embolização, promover o risco de causar um novo local de vazamento, a presença de múltiplos vasos nutridores e a possível necessidade de uma nova intervenção aumentam ainda mais a cautela para utilização desta técnica ${ }^{5}$.

Neste trabalho observamos, ao estudo com tomografia computadorizada helicoidal, que o paciente evoluiu com diminuição do diâmetro do aneurisma após a ligadura da artéria mesentérica inferior por via videolaparoscópica.

A ligadura videolaparoscópica pode ser uma boa opção para o tratamento do endoleak causado pela artéria mesentérica inferior, com o objetivo de proporcionar ao paciente uma recuperação pós-operatória rápida, tempo de internação hospitalar pequeno, discreta cicatriz, e retorno rápido do paciente às suas atividades normais.

\section{ABSTRACT \\ Videolaparoscopy has been widely used in the treatment of pathologies as cholelithiasis, appendicitis and adrenal tumor. Nowadays, has also been used to treat type II endoleaks after endovascular repair of abdominal aortic aneurysms. The goal of this work is to report one case of inferior mesenteric artery endoleak treated by videolaparoscopy.}

Key Words: Aortic aneurysm, abdominal; Surgery, laparoscopic; Aorta, grafts and prostheses; Aorta, interventional procedures; Stents and prostheses. 


\section{REFERÊNCIAS}

1. Baum RA, Carpenter JP, Tuite CM et al. Diagnosis and Treatment of Inferior Mesenteric Arterial Endoleaks after Endovascular Repair of Abdominal Aortic Aneurysms. Radiology 2000;215:409-413.

2. Gilling-Smith, G, Brennan J, Harris P et al. Endotension after Endovascular Aneurysm Repair: Definition, Classification, and Strategies for Surveillance and Intervention. J. Endovasc. Surg. 1999;6:305-307.

3. Görich J, Rilinger N, Sokiranski, R et al. Leakages after Endovascular Repair of Aortic Aneurysms: Classification Based on Findings at CT, Angiography, and Radiography. Radiology 1999;213:767-772.

4. Espinosa G, Murad H, Silva LF et al. Acompanhamento Tomográfico de Aneurisma de Aorta Abdominal durante 32 meses, após Implante de Endoprótese. Rev. Angiol. Cir. Vasc. 2000;9:59-63.
5. Wisselink W, Cuesta MA, Berends F.J et al. Retroperitoneal Endoscopic Ligation of Lumbar and Inferior Mesenteric Arteries as a Treatment of Persistent Endoleak after Endoluminal Aortic Aneurysm Repair. J. Vasc. Surg. 2000;31:1240-1244.

Endereço para correspondência:

Gaudencio Espinosa

Praia de Icaraí, 113/801

24230-001 — Niterói-RJ 